The group $2 M_{2}\left(N_{1} \leftarrow N_{2}\right)$ was located at $\nu=18000$, by following the extension of the irregular doublet law given by Saha and Kichlu, and by treating the spectrum as the mean between those of $\mathrm{P}^{+}$and $\mathrm{Cl}^{+}$ both recently analysed by Bowen. An examination of the tables of Eder and Valenta revealed a group of strong lines in this region.

A further clue was obtained from the fact that of these groups of lines only $\lambda 5454$ and $\lambda 5033$ were traced by Lockyer in the spectrum of Rigel. Hence these two lines were regarded as the strongest lines of the group $2 M_{2} N_{1} \longleftarrow 2 M_{2} N_{2}$, namely, as ${ }^{4} P_{3}-{ }^{4} D_{4}$ and ${ }^{4} P_{3}-{ }^{4} \bar{P}_{3}$. Both of these multiplets have been discovered, $\Delta p_{12}$ and $\Delta p_{23}$ of $2 M_{2} N_{1}$ being respectively 270 and 437 . The $4 D$ differences are 151,257 , and 366.

With these multiplets as clues, the other strong groups identified by Lockyer at $\lambda 4142-\lambda 4174$ with some lines in the spectrum of Rigel could be assigned to the ${ }^{4} D-{ }^{4} D,{ }^{4} P-{ }^{4} P$ groups of the transition $2 M_{2} M_{3} \leftarrow 2 M_{2} N_{2}$. They are not reproduced here.

Patna and Allahabad, D. K. BHATTACHARYYA. May 8.

\section{Fluorescence of Mercury Vapour under Low Excitation.}

IT has, I believe, been generally supposed that the green band fluorescence of mercury vapour cannot be excited by optical frequencies much less than that of the mercury resonance line. Houtermans, working in Franck's laboratory, has put forward the view that this green fluorescence results from the excitation of the molecule to the $2^{3} \mathrm{P}_{1}$ state, differing but little in energy from the corresponding state of the atom.

I have recently found, however, that this high frequency is not necessary to stimulate the green fluorescence. The most effective arrangement tried so far is to focus an iron or copper arc, or an ' atmospheric' mercury lamp, on a bulb containing mercury at atmospheric pressure. A filter consisting of a bromine cell and a sheet of blue uviol glass is interposed to cut off visual light. Under these conditions a bright green track is seen marking out the path of the rays in the vapour.

The shortest mercury wave-length that can get freely through the blue uviol glass is $\lambda 3125$, and experiments with a monochromator have shown that this line excites the fluorescence. The strong line at $\lambda 3650$ is unable to do so.

These experiments are being developed in various directions, and will be published more fully in due course.

Terling Place,

RAYLEIGH.

Chelmsford, Essex,

Aug. 4.

\section{Radium in Cancer.}

In Nature of Aug. 4, the writer of the article on Cancer Problems, after stating that substantial practical advance has been made in treatment by radium, concludes by saying, "At the same time there is no justification for any talk about surgery being eliminated."

At the International Conference in question there were discussions upon the relative value of surgery and radiology in the treatment of cancer in four sites of the body. At these discussions four British surgeons spoke in the following terms: one advocated the use of radium in operable breast cancer; one stated that he had given up the Wertheim operation for cancer of the cervix uteri in favour of radium; one described a series of cases of cancer of the tongue where the primary growths were treated by radium in order to avoid excision; and one detailed a method of radium treatment of cancer of the rectum, in operable and inoperable cases during the last two years.

When statements like these are made by surgeons themselves, would it not be more correct to say that owing to the advances in radium-therapy there is some justification for believing that in certain sites of cancer, radium may with advantage replace surgery?

The Middlesex Hospital, W.1, Aug. 6.

Prof. Russ gives the more correct description of the proceedings of the Congress: the lay press translated them with inaccurate exaggeration.

The Writer of the Article.

\section{The Spectrum of Tribly Ionised Antimony, Sb IV}

IN the case of the isoelectronic system Cd I, In II, Sn III, Sb IV, the X-ray doublet laws have been found to apply even though these spectra consist of singlets and triplets. Continuing our previous work on In II and Sn III, we have been able to identify several groups in the spectrum of $\mathrm{Sb}$ IV. These are a ${ }^{3} P^{3} S$ multiplet, lying between $805 \mathrm{~A}$. and $861 \mathrm{~A}$., a very strong ${ }^{3} P^{3} D$ multiplet, lying between $873 \mathrm{~A}$. and $940 \mathrm{~A}$., a ${ }^{3} D^{3} \mathrm{~F}$ multiplet, lying between $2077 \mathrm{~A}$. and $2113 \mathrm{~A}$., and a possible ${ }^{3} P^{3} P^{\prime}$ group between $1051 \mathrm{~A}$. and $1193 \mathrm{~A}$. The first ${ }^{3} P$ separations are 5854 and $2261 \mathrm{~cm}^{-1}$. In addition to these, $a{ }^{1} S^{3} P$ line and ${ }^{1} S^{1} P$ line give an estimate of $340000 \mathrm{~cm}^{-1}$ for the lowest, ${ }^{1} S$, level corresponding to an ionisation potential of about 42 volts.

In addition, second members of series have been identified in In II, and we are now looking for additional combinations in Sn III and Sb IV. A complete report will be published elsewhere.

Columbus, Ohio.

Edmonton, Alberta.

\section{J. B. GREEN.}

R. J. LANG.

The Corpus Luteum and the Cause of Birth.

As I pointed out in the article referred to by Prof. Thomson Flynn in NATURE of June 30, p. 1020 (Biol. Rev., 2, 129; 1927), parturition is certainly due to several factors, of which the decline of the corpus luteum is probably one. Moreover, the enlargement of the pregnant uterus is also due to several factors. In the case of the sterile uterine horn in Bettonqia, the partial regression of the corpus luteum in the absence of the fotus may have been the main factor in the uterine involution. In the case of the other horn the enlarged condition of the uterine wall and the contained fœtus involve further factors in the continuation of pregnancy, and it may be that in their presence the regression of the corpus luteum was not sufficiently advanced to admit of the occurrence of birth at the time of the commencement of involution in the non-pregnant horn; that is to say, in order that parturition may occur, it may be necessary not only for the corpus luteum to be in a state of marked regression, but also for the uterine horn and contained foetuses to be in a certain condition of development.

July 15 .

No. 3068 , VoL. 122] 